\title{
SITUACIÓN LABORAL DE LAS MUJERES CON DISCAPACIDAD FÍSICA Y SENSORIAL EN LA PROVINCIA DE ALICANTE
}

Recibido: 05/11/2008

\author{
CARMEN MAÑAS VIEJO \\ Universidad de Alicante \\ ALMUdENA INIESTA MARTíNEZ \\ Universidad de Alicante
}

Aceptado: 03/03/2009

\section{Introducción}

En este artículo realizamos un análisis y evaluación con perspectiva de género de la situación laboral de las mujeres con discapacidad física y sensorial en la provincia de Alicante. Hemos diferenciado entre dos ámbitos de estudio: el privado (desarrollo personal, familia y relaciones personales) y el público (barreras, educación, trabajo, situación e imagen social y movimiento asociativo).

Pretendemos identificar aquellas variables que estén generando una situación de doble discriminación, por razón de género y discapacidad, en el ámbito laboral. Queremos poner voz a las experiencias de vida de las mujeres con situación discapacitante. Nuestra intención última es aportar información necesaria, que pueda ser tenida en cuenta a la hora de programar una acción política determinada de cuya ejecución derive una intervención adecuada, que favorezca y optimice su integración y consiguiente normalización ${ }^{1}$.

Nuestra metodología tiene un carácter mixto, tanto cuantitativo como cualitativo, y de campo, con una finalidad: poder conocer cuáles son los

1. Nos parece muy interesante la reflexión de P. Riu sobre la normalización. «Una perspectiva de género desde la no estandarización». En Shum, G y Conde, A. (Eds). Las olvidadas: Mujeres en riesgo de exclusión social. Huelva, Universidad, 2006, pp. 186-246. 
episodios biográficos tanto sociales como personales que han contribuido y que subyacen en el estado y la situación actual de las participantes, con un alcance temporal retrospectivo y prospectivo. Las historias de vida que se van a estudiar van a ser explicadas y narradas por tres grupos de mujeres con discapacidades de la provincia de Alicante, diferenciadas en tres campos: discapacidad física, deficiencia visual y deficiencia auditiva. De los cuales se infiere la particularidad y significatividad de cada mujer específica.

Las mujeres participantes en el estudio ${ }^{2}$ tienen edades comprendidas entre los 15 y los 50 años, sus discapacidades pueden ser sobrevenidas o congénitas. Para el análisis de los datos hemos seleccionado el programa AQUAD Five para Windows ${ }^{3}$ con el que hemos podido realizar interpretaciones, categorizaciones y codificaciones en base al discurso de las mismas de las mujeres, sin necesidad previa de crear un sistema de categorización prediseñado mediante teorías.

\section{Fundamentación teórica}

En nuestra investigación necesitamos posicionarnos teóricamente frente a dos constructos: discapacidad y género, mujeres con discapacidad. Construcción de dos parámetros que hasta hace muy pocos años no se habían unido en estudio. Es en estos últimos años ${ }^{4}$ cuando ha empezado a preocupar la relación e influencia directa que tiene el uno sobre el otro, al asumir que el hecho de ser mujer agrava y condiciona en un mayor grado la situación, realidad y problemática de la discapacidad. Observamos que es en la actualidad cuando empieza a inquietar que el contexto discapacitador se vertebra actuando desde dos razones: género y discapacidad. Nos encontramos por primera vez plasmada esta realidad en la Cuarta Conferencia Mundial sobre Mujer en Beijing 1995, donde los gobiernos se comprometen a cumplir las previsiones que se han realizado a favor de la mujer con discapacidad. Esto significa que se han comprometido a hacer lo necesario para que las recomendaciones que afectan a las mujeres con discapacidad se hagan realidad. Desde nuestra investigación

2. El estudio en el que nos basamos es la Tesis doctoral defendida por Almudena Iniesta Martínez en la Universidad de Alicante en julio del 2009, titulada: "Análisis y evaluación desde una perspectiva de género de la situación de las mujeres con discapacidad física y sensorial en la provincia de Alicante».

3. AQUAD Five para Windows (Huber, Günter de la Universidad de Tubinga, 1990-2003).

4. En este sentido podemos señalar dos fechas significativas: 1995 Conferencia de Beijing, que constituye un punto de inflexión sobre la consideración del hecho de ser mujer y sus consecuencias sobre la discapacidad, y 2003, año del Primer Congreso Internacional sobre el género y la discapacidad realizado en la ciudad de Valencia. 
creemos esencial clarificar el concepto de mujer con discapacidad, que incluye a mujeres con cualquier clase de discapacidad, mujeres con deficiencias físicas, auditivas, visuales o mentales, sean estas visibles o no, mujeres con enfermedades crónicas como la diabetes, enfermedades renales y cardiacas, epilepsia, VIH/sida, o enfermedades que afecta sobre todo a la mujeres, tales como fibromialgia, artritis, cáncer de mama, lupus y osteoporosis. El término también incluye a las niñas y mujeres con discapacidad de cualquier edad, residentes en áreas rurales o urbanas, sin importar la gravedad, ni sus preferencias sexuales o su entorno cultural, vivan integradas en la comunidad o en instituciones ${ }^{5}$.

El marco teórico en el que más cómodas nos sentimos al estudiar el constructo género y discapacidad es el modelo social de discapacidad, pero no de manera exclusiva. Compartimos con este modelo la necesidad de investigar y analizar desde las propias experiencias de las mujeres con discapacidad, porque si no se imponen las propias definiciones y perspectivas de las mujeres con discapacidad, el mundo no discapacitado seguirá haciéndolo por ellas, seguirá alienándolas y descalificándolas. Se han obviado las experiencias de las mujeres con discapacidad; urge introducirlas en la agenda política. Tener en cuenta su propia experiencia, eliminar los miedos a poder hablar del dolor, de las perdidas individuales... Su subjetividad es el objetivo que da origen al modelo social de la discapacidad.

Con el modelo social surgen nuevos términos como: «discapacitacion», «no-estándar» y también, entre otros, «barreras discapacitantes», que aluden a los obstáculos externos, impuestos por la sociedad estándar. El modelo social permite escribir sobre la experiencia personal del cuerpo y de la mente ${ }^{6}$. Las personas con discapacidad no son responsables de todas las dificultades que sufren; es su entorno el que crea las barreras sociales. No obviaremos en nuestro estudio la experiencia subjetiva de cada mujer con su cuerpo.

Recriminamos al modelo médico su concepción de individuo enfer$\mathrm{mo}^{7}$, de identidad enferma, pero no lo rechazamos. Nos acercamos, en este sentido, mucho más al nuevo modelo social crítico $^{8}$, que intenta mirar a la

5. ZIJDEL, Lidia. «Manifiesto de las mujeres con discapacidad en Europa». En García, N y Cuesta, Z (Eds.) Minusvalía Una aproximación al tema de la discapacidad. IMSERSO, Madrid, 2000, pp. 430-431.

6. MORRIS, Jenny. Encuentros con desconocidas. Madrid, Narcea, 1996.

7. Verdugo, M. A., De Borja, F. y De Urries, J. (Coords.). Hacia una nueva concepción de la discapacidad. Universidad de Salamanca, 1999; CROW, Liz. «Nuestra vida en su totalidad: renovación del modelo social de discapacidad». En MorRIS, Jenny. Encuentros con desconocidas. Madrid, Narcea, 1996, pp. 22-25.

8. CROW, Liz. Op.cit. 
discapacidad como una identidad más de las múltiples de las que disfruta el ser humano. Este nuevo modelo crítico nos aporta una visión más amplia de la discapacidad, a la que ve no sólo como un hecho social, sino como una identidad propia del ser discapacitada/o, que hace ineludible la intervención de las propias actoras en su desarrollo, eludiendo de forma consciente la victimización y potenciando las otras identidades, las no discapacitadas.

Contemplamos la heterogeneidad de las diferentes trayectorias vitales de la población con discapacidades. No existe una homogeneidad en el grupo que permita medir todas las discapacidades con un número porcentual, debemos atender a la significatividad de cada persona con discapacidad.

En España los datos cuantitativos acerca de las mujeres con discapacidad son casi nulos, nos centramos en la Encuesta sobre Discapacidades, Deficiencias y Estado de Salud de $1999^{9}$, (en adelante EDDES), que sigue siendo, en la actualidad, la referencia para cualquier investigación relacionada con la discapacidad. Según la EDDES, hay más de 3,5 millones de personas con alguna deficiencia o discapacidad, lo que supone un $9 \%$ de la población. De éste, el porcentaje de mujeres con discapacidad es mayor en un 58 por 100 que el de los hombres.

En cuanto al empleo, que como sabemos es el instrumento por el que la mayoría de las personas obtenemos recursos para poder vivir, además de ser un espacio de participación social y desarrollo personal ${ }^{10}$, la tasa de paro

9. La Encuesta sobre Discapacidades, Deficiencias y Estado de Salud, realizada en 1999 por el Instituto Nacional de Estadística con la colaboración técnica y financiera del Instituto de Migraciones y Servicios Sociales y de la Fundación ONCE, es una gran operación estadística en forma de macroencuesta (con una muestra efectiva de 70.500 viviendas, lo que supone haber recabado información sobre unas 218.000 personas en toda España) orientada a cubrir las necesidades de información sobre los fenómenos de la discapacidad, la dependencia, el envejecimiento de la población y el estado de salud de la población residente en España.

Los resultados preliminares de la Encuesta sobre Discapacidades, Deficiencias y Estado de Salud fueron apareciendo durante 2001, cuando habían transcurrido casi quince años desde la publicación de los resultados de la anterior encuesta, realizada en 1986. La publicación de los resultados detallados se ha producido en el mes de junio de 2002, fecha en la que también se han puesto a disposición de los investigadores los microdatos de la misma.

10. El 10 de julio de 2009 el Consejo de Ministros aprobó el III Plan de Acción para las Personas con Discapacidad, que establece la estrategia del Gobierno para esta Legislatura en materia de discapacidad. De acuerdo con los principios de la Ley de Igualdad de Oportunidades, no Discriminación y Accesibilidad Universal, de 2 de diciembre de 2003, el III Plan de Acción se ha elaborado en colaboración con el Comité Español de Representantes de Personas con Discapacidad (CERMI). La aplicación del principio de transversalidad, afecta a todas las políticas. Además, actúa como plan director u orientador de las políticas de las Comunidades Autónomas y las ONG. 
entre los 15 y 50 años es, según la Encuesta de Población Activa ${ }^{11}$ (EPA), del 25,8 por 100. Mientras que la tasa de paro de la población normalizada, en el mismo trimestre que se realizó el estudio, era, del 15,73 por 100. La tasa de paro en mujeres con discapacidad es del 32,7 por 100 , claramente superior a la masculina que supone más del $21 \%$ y mayor también que la tasa de paro laboral de las mujeres estándar que supera el 23\%. De los datos reflejados podemos concluir que de entre los sectores de la población con mayor dificultad de acceso a un puesto de trabajo se encuentran las mujeres con discapacidad.

En España, en general, y en Alicante en particular, la incorporación de las mujeres con discapacidad al mercado de trabajo ha sido un fenómeno relativamente reciente, al que aún le queda un larguísimo camino que recorrer, pero que no hay que olvidar que está en marcha. Naturalmente son muchos los factores que influyen de forma determinante en la situación laboral en la que se encuentran las mujeres que tienen discapacidad, señalamos el sistema educativo y el entorno cultural. Contamos con un sistema educativo escasamente adaptado a la discapacidad con aún graves deficiencias en accesibilidad física, escasas adaptaciones curriculares y en general escasez de recursos específicos que mantienen a las personas con discapacidad en la sombra. Y unido a ello un entorno generosamente sexista, estereotipado y con escaso saber, que apenas comienza a plantearse la necesidad de entrenar sus capacidades para conquistar el espacio público, para acceder a un puesto de trabajo, para desarrollarse personalmente en igualdad de oportunidades; en definitiva, un entorno que como expone Peláez ${ }^{12}$, sigue creyendo que Ellas merecen menos.

\section{Cuestiones de la investigación}

Partimos de la hipótesis que las mujeres con discapacidad física y sensorial están sumidas en una doble discriminación por razón de género y discapacidad y, por ello, encuentran multitud de obstáculos en su vida tanto pública como privada.

Para operativizar nuestro objeto de estudio lo hemos delimitado, mediante objetivos específicos, a partir de los ámbitos de estudio propuestos, público y privado. Dentro de ellos, el contenido lo hemos estructurado en diferentes áreas: Privado: Aspectos Personales, Familia y Barreras discapacitadoras

11. La Encuesta de Población Activa (EPA) es un estudio estadístico de información sobre la actividad de la población distribuidos por el Instituto Nacional de Estadística.

12. Peláez NarvaÉz, A. M. Maternidad y discapacidad. Madrid, Cermi, Barclays, 2009, p. 74.

Feminismo/s 13, junio 2009, pp. 153-170 
y/o discapacitantes; y Público: Educación, Situación e Imagen Social, Trabajo, Movimiento Asociativo y Barreras discapacitadoras y/o discapacitantes.

Este trabajo que presentamos se centra en conocer y analizar las dificultades con las que se han encontrado en el proceso de acceso a un empleo tanto en el ámbito privado y familiar como en el público. Queremos conocer su percepción respecto a cuestiones como los prejuicios de los empleadores y el estigma social.

Consideramos que su participación proporciona visualización y desmitificación a sus vidas. Profundizamos en sus vivencias, en sus experiencias en la busca de empleo. Una realidad contada en primera persona.

\section{Diseño metodológico}

La estrategia metodológica que hemos llevado a cabo en este estudio está enmarcada dentro de la disciplina de investigación social que, como define Sierra $^{13}$, es el proceso de aplicación del método y técnicas científicas a situaciones y problemas concretos en el área de la realidad social, para hallar respuesta a ellos y adquirir nuevos conocimientos.

La finalidad en nuestra investigación es el conocimiento acerca del género y de la discapacidad, conocer de qué manera influye la confluencia de este binomio en las mujeres que tienen discapacidad física y sensorial en la provincia de Alicante. Analizamos tanto retrospectiva como prospectivamente la situación individual de cada mujer con discapacidad que participa en nuestro trabajo, quedando éste enfocado a las investigaciones sociales explicativas y exploratorias, de amplitud microsociológica. Las fuentes utilizadas han sido tanto de primera mano como secundarias, configurando un modelo mixto de recogida de información en una investigación de carácter fundamentalmente cualitativo.

Fue necesaria la elaboración de un registro propio para poder seleccionar una muestra representativa de la población de mujeres con discapacidad física y sensorial de entre 15 y 50 años que pertenecen al suroeste de la provincia de Alicante (Elche, Alicante, Santa Pola, Denia, Pego y Orihuela). Para ello, hemos contado con la colaboración de diferentes instituciones sociales, federaciones de personas con discapacidad, tanto física como sensorial, y asociaciones, todas ellas de la provincia de Alicante.

Finalmente en nuestra investigación y, siempre de manera voluntaria, han participado 50 mujeres:

13. SierRA, R. Técnicas de investigación social. Teoría y ejercicios. (Decimocuarta Edición). Madrid, Thomson, 2003. 
18 Mujeres con Discapacidad Física.

16 Mujeres con Discapacidad Visual o Ciegas.

16 Mujeres con Discapacidad Auditiva o Sordas.

Cuadrol

\begin{tabular}{|c|c|c|}
\hline \multicolumn{2}{|c|}{$\begin{array}{c}\text { TIPO DISCAPACIDAD } \\
\text { Códigos }\end{array}$} & $\begin{array}{c}\text { Mujeres con } \\
\text { Discapacidad }\end{array}$ \\
\hline Auditiva & /SORD & $34,70 \%$ \\
\hline Física & /FIS & $32.66 \%$ \\
\hline Visual & /VIS & $32.66 \%$ \\
\hline
\end{tabular}

La presencia de mujeres con discapacidad física y sensorial es casi homogénea en los tres grupos, el porcentaje de mujeres con discapacidad auditiva es un $(34.70 \%)$, es $2 \%$ mayor respecto al de las mujeres con discapacidad física y visual que es idéntico porcentaje, (32.66\%).

Pretendemos formar un circulo abierto, en el que las colaboradoras puedan reflexionar y re-construir su propia narrativa. Y ello, desde las reflexiones de sus vidas en los diferentes ámbitos público y privado, atendiendo al pasado, al presente y mirando hacia el futuro.

Este estudio lo hemos llevado a cabo realizando entrevistas en profundidad de carácter semi-estructurado. El ámbito laboral ha sido estudiado utilizando el siguiente guión:

1.- Recogida de la información estructurada. Las variables de clasificación con las que trabajamos son: edad, momento de aparición de la discapacidad, nivel académico, situación laboral. Se trata de un total de 36 ítems que están formulados para obtener información cuantitativa.

Cuadro 2

Código descriptivo y demográfico: Situación laboral.

\begin{tabular}{|c|c|c|}
\hline \multicolumn{2}{|c|}{$\begin{array}{c}\text { SITUACIÓN LABORAL } \\
\text { Código }\end{array}$} & $\begin{array}{l}\text { Mujeres con } \\
\text { Discapacidad }\end{array}$ \\
\hline Paro & / PARO & $32,65 \%$ \\
\hline Trabaja & /TRBJA & $30,61 \%$ \\
\hline P.N.C & / P.N.C & $28,48 \%$ \\
\hline P.C & / P.C & $4,08 \%$ \\
\hline Estudia & /ESTD & $8,16 \%$ \\
\hline
\end{tabular}

Feminismo/s 13, junio 2009, pp. 153-170 
El tramo de mayor representación en este código pertenece a las mujeres con discapacidad física y sensorial que se encuentran en una situación de paro (32.65\%), seguido por el grupo de mujeres que se encuentran trabajando, (30.61\%) y el nivel más bajo de porcentaje son las de mujeres que tiene una pensión contributiva (4.08\%).

2. - Guión entrevista en profundidad, en este caso sobre el empleo. Considerándolo parte integrante del proceso de independencia, autoestima y reconocimiento social, se hace esencial que las mujeres con discapacidad reciban una formación que les permita acceder con oportunidades al mercado de trabajo. En este sentido queremos saber cuáles han sido sus oportunidades de formación, cómo las han vivido, qué experiencias les han resultado significativas y estimulantes y cuáles les han perjudicado, qué tareas les asignan y cuáles han podido elegir, con qué bagaje cuentan para afrontar su adecuación al mundo laboral y cuál ha sido, en definitiva, su experiencia; y también, de manera importante, sus perspectivas. Y todo ello, naturalmente, sin obviar ni la subjetividad ni la intersubjetividad ${ }^{14}$ que impregna tanto la vida privada como la pública. Huimos decididamente de cualquier tipo de construcción de escalas que las diferencie frente a las mujeres sin discapacidad.

Transcribimos a continuación las preguntas guía que se han realizado para estudiar en profundidad la experiencia de cada una de ellas en relación con el empleo, tanto desde el ámbito privado como desde el público.

- ¿Crees que los empresarios ponen más trabas a la hora de contratar a mujeres con discapacidad que a hombres? ¿Por qué?

- ¿Las condiciones laborales, piensas que son las mismas: sueldo, contrato...?

- ¿Qué empleos son los más solicitados por las mujeres con discapacidad? ¿Y los hombres con discapacidad?

- ¿Piensas que existe desigualdad de condiciones entre hombres y mujeres con discapacidad a la hora de encontrar empleo?

- ¿Has realizado cursos de formación o reciclaje en tu empresa?

- ¿Piensas que las barreras arquitectónicas, comunicativas, mentales, etc. son un problema grave para el logro y desarrollo de un trabajo? ¿Y el hecho de ser Mujer?

La recogida de datos la realizamos adaptándonos al contexto y situación de cada participante mediante técnicas que sirvan de base y de guía para el desarrollo de sus narrativas. Valoramos, determinamos y ligamos las condiciones y especificidades de este proceso de investigación a las características

14. HeRnANDO, A. La construcción de la subjetividad femenina. Madrid, Universidad Complutense, Instituto de investigaciones feministas, 2000. 
individuales de las participantes, contribuyendo a crear un clima de colaboración y empatía.

El análisis exhaustivo de las narrativas lo interpretamos ${ }^{15}$ mediante la elaboración de categorías demográficas y descriptivas de las unidades significativas de sus historias de vida. Para ello hemos utilizado el programa AQUAD Five $^{16}$ que permite hacer flexible y aportar elasticidad al proceso de interpretación, categorización y codificación de estas unidades significativas. Así podemos reiniciar, añadir, cambiar y eliminar códigos durante el desarrollo de la investigación, lo que aporta al estudio un carácter de continuo crecimiento y evolución.

Dentro del ámbito laboral hemos interpretado los siguientes códigos:

- Mujeres relegadas al desempeño de trabajo no mercantilizado, en el que hemos analizado, dada su importancia, dos subcódigos muy cercanos entre sí: Escasa Formación y Rol Femenino.

- Empleos feminizados.

- Cargas de estigmas y prejuicios sociales acerca de las mujeres con discapacidad.

- Inferioridad de oportunidades.

- Discriminación por razón de género.

\section{Discusión de los resultados}

5.1. Discusión de los resultados del código: Mujeres relegadas al desempeño del trabajo no mercantilizado

Fundamentalmente, a las mujeres con discapacidad física y sensorial se les designan tareas del ámbito privado, el cual no está valorado. Desempeñan el rol que les han adjudicado como mujeres y, en especial, como mujeres con discapacidad, asumiendo con bastante resignación valores y creencias patriarcales y estandarizadas. Esta situación, en general, se vincula a la escasa cualificación académica y a la falta de expectativas personales y laborales.

Ajustamos este código en dos subcódigos muy cercanos entre sí: Escasa Formación y Rol Femenino.

15. El esquema de códigos, metacódigos y subcódigos que se enmarca dentro de este ámbito, surge tras el análisis exhaustivo de las narrativas de las mujeres con discapacidad que han participado en la investigación y con la colaboración de un equipo experto de profesores que han actuado de jueces para la validez de los códigos tanto demográficos y descriptivos

16. Huber, G L. Fernández, G. Lorenzo, O y Herrera, L. Análisis de datos cualitativos con Aquad cinco para Windows. Granada, Grupo editorial universitario, 2001. 


\section{Cuadro 3}

\begin{tabular}{|c|c|c|}
\hline \multirow{2}{*}{$\begin{array}{c}\text { Mujer relegada al desempeño } \\
\text { del trabajo no mercantilizado }\end{array}$} & Porcentajes & Frecuencias \\
\cline { 2 - 3 } & $12.24 \%$ & 0.12 \\
\hline Escasa formación & $71.36 \%$ & 0.71 \\
\hline Rol femenino & & \\
\hline
\end{tabular}

\subsubsection{Discusión de los resultados del subcódigo: Escasa formación}

Las mujeres con discapacidad física y sensorial, en muchos casos, no tienen formación suficiente ${ }^{17}$, lo que les dificulta enormemente el acceso a un empleo remunerado, y si lo encuentran, en la mayoría de las situaciones, son empleos poco valorados socialmente. En sus narrativas expresan cómo no se considera necesaria y esencial la formación de las mujeres con discapacidad. Sin embargo, en nuestro actual contexto sociolaboral, nadie podría argumentar con rigor esta consideración para cualquier otra persona, incluidos los hombres con discapacidad. Lamentablemente el porcentaje de aparición de este subcódigo es bajo $12,24 \%$, son pocas las mujeres entrevistadas que verbalizan cómo la escasa formación que tienen les perjudica en su inserción laboral y cómo el mismo hecho consolida sus vidas dentro del ámbito privado:

... la mujer le falta estudiar y la mujer si no es ama de casa (sord/006)

...que se adapten los centros educativos, pero la mujer discapacitada sí se ha encontrado con menos oportunidades de estudiar y eso ha hecho que tengan los peores trabajos o que sólo estén en la casa [...] trabajos profesionales muy pocos, porque antiguamente no se estudiaba, las mujeres estaban muy marginadas, los únicos que estudiaban eran los que tenían una familia que les apoyaba (sord/001)

\subsubsection{Discusión de los resultados del subcódigo: Rol femenino.}

El porcentaje de frecuencia de este subcódigo es bastante significativo, ya que el $77,36 \%$ de las mujeres con discapacidad física y sensorial afirman asumir el rol que ha sido asignado a su género, sus acciones y sus responsabilidades. En definitiva, interiorizan que podrán llegar a desempeñar labores de cuidadoras, madres y esposas, pero nunca como es debido, y que, para ello, no es necesario tener nivel educativo ${ }^{18}$. Asumen con resignación que la consecuencia

17. HERNÁNDEZ, I. (coor.). Situación laboral de las mujeres con discapacidad. Madrid, Instituto de la mujer, 2005.

18. HERNÁNDEZ, I. Op cit. 
natural de ser mujer y tener discapacidad es no acceder a un puesto de trabajo remunerado.

Exponen la dificultad de tener la responsabilidad del hogar, del cuidado de las/os hijas/os y se sienten incapaces de poderlo compaginar con un trabajo remunerado, lo expresan con desanimo y desilusión. Afirman sentirse poco reconocidas a nivel familiar, laboral y social.

Ya te he comentado antes, el hecho de ser mujer yo creo que ya supone estar por debajo un poco de los hombres, mírame a mí, ama de casa y cuidando de mis hijas, si tienes un problema de visión como yo, es más difícil buscar empleo porque tienes niñas que tienes que seguir cuidando y encima no ves, quién te va a querer habiendo tanta competencia para trabajar, es duro tener una deficiencia (Vis016)

...porque una mujer tiene que estar en casa atender al marido, tiene que saber guisar y tiene que ser seductora, pero tú, mujer con discapacidad no eres bonita, no eres seductora, entonces qué vas hacer, ya eres la que cuida a tus padres cuando son mayores (Fis010)

...están las que se casan muy jóvenes y te dicen que no pueden estudiar que tienen que cuidar a sus hijos, a su marido y a lo mejor tienen 20 años, o les dices «venid a la conferencia que van a dar en la asociación con intérpretes» y no vienen porque no les interesa aprender, se han acostumbrado a otra vida, la de ama de casa (Sord003)

\subsection{Discusión de los resultados del código: Empleos feminizados}

Las mujeres con discapacidad física y sensorial, en su mayoría, ejercen trabajos que se consideran exclusivos de su género y socialmente menos válidos. Son trabajos relacionados con las tareas del hogar pero realizados en el ámbito público. Por ejemplo, empleadas en empresas de limpieza, lavanderías... En general, son trabajos donde la fuerza física no prima, asignados a su género y fortalecidos según ese innato saber que las mujeres tienen para realizar las tareas que imperan como naturales a su género.

\section{Cuadro 4}

\begin{tabular}{|l|c|}
\hline \multirow{4}{*}{ Empleos feminizados } & Media de \\
& frecuencia \\
& 1.32. \\
\cline { 2 - 2 } & Porcentaje \\
& $132.65 \%$ \\
\hline
\end{tabular}

Feminismo/s 13, junio 2009, pp. 153-170 
El porcentaje de frecuencia de este código es muy significativo, dada la elevada presencia que tiene en las narrativas de las mujeres entrevistadas. Ellas declaran estar clasificadas en un tipo de empleos en los que el rol asignado a la mujer es la norma, están ligadas a trabajos con ínfima valoración social, destinados exclusivamente a las mujeres con discapacidad física y sensorial.

Yo creo que de las discapacitadas psíquicas es de limpieza, lavandería, de limpieza de arreglar flores y luego, de las mujeres discapacitadas físicas y ciegas pues de trabajo social, de oficina, funcionaria, secretaria algunas profesores (Fis001)

Los de limpieza, lavanderías, en la Once como vendedor del cupón puede que también algunos en el calzado (Vis014)

Los de limpieza para las mujeres casi siempre, la mujeres sordas que trabajan lo hacen en la limpieza, los hombres más cosas en trabajos que no cogen a mujeres (Sord004)

\subsection{Discusión de los resultados del código: Cargas de estigmas y prejuicios} sociales acerca de la mujer con discapacidad

La percepción social que se tiene de las mujeres con discapacidad como personas con escaso potencial, necesitadas de ayudas y de prestaciones sociales, siendo no competitivas dentro del mercado de trabajo, afecta a las posibilidades que desde la sociedad se les ofrecen. Como consecuencia de los valores y creencias sociales negativos acerca de sus posibilidades y capacidades, las etiquetan, y ello constituye un nuevo obstáculo, demasiadas veces insalvable, en el logro de un empleo.

\section{Cuadro 5}

\begin{tabular}{|c|c|}
\hline & Media de \\
Cargas de estigmas & frecuencia \\
y prejuicios sociales & 0.53 \\
\cline { 2 - 2 } acerca de la mujer con & Porcentaje \\
\cline { 2 - 2 } discapacidad & $65.30 \%$ \\
\hline
\end{tabular}

El porcentaje de presencia de este código es muy significativo, las mujeres con discapacidad física y sensorial consideran que, en general, las valoraciones negativas respecto a ellas están presentes en la sociedad. Creen que estas valoraciones y creencias negativas tienen su origen en el desconocimiento, son juzgadas por el hecho de ser mujeres con discapacidad, que no cumplen con el canon mínimo que como mujeres se espera de ellas: belleza y salud. No 
sienten que se les dé la oportunidad de expresar, desarrollar y proyectar sus capacidades.

donde más problemas he tenido ha sido en el trabajo, he ido a buscar trabajo y sí, he tenido la pega de ser sorda, a lo mejor he tenido que ir alguna parte no sabría como explicarte, me he sentido discriminada un poco (Sord009)

- Existen barreras externas que frenan la igualdad laboral y un sentimiento de rechazo:

En muchos casos, sí, porque el físico cuenta mucho y si ven a una mujer bien la prefieren sin saber si la mujer con discapacidad lo puede hacer mejor o igual, a mí me ha pasado en algún trabajo que me han desvalorizado por mi deficiencia visual [...] yo lo he vivido en mi propia persona, yo trabajaba cuidando una niña y yo lo hacía todo muy bien pero mi jefe sólo me había visto con lentillas, nunca con gafas y el día que se enteró de lo que a mí me pasaba y vio las gafas y me dijo que yo no podía cuidar a una niña tan pequeña que se me podía perder, me dijo un montón de cosas que no tienen sentido. (Vis008)

Yo a la hora de buscar empleo más que por mi minusvalía ha sido la dificultad de mi estatura, como yo la enfermedad la tuve tan pequeñita pues no me dejó crecer más y por eso soy tan pequeña, el hecho de ser tan bajita a la hora de conseguir un trabajo me dificulta, porque me han rechazado, en muchos trabajos, por la estatura, en algunos trabajos me lo han dicho y en otros lo he entendido así porque era evidente. (Fis005)

En síntesis, en un alto porcentaje (65.30\%), hallamos narrativas alusivas a las cargas de estigmas y prejuicios sociales acerca de la mujer con discapacidad, que influye de forma negativa en la inserción laboral.

\subsection{Discusión de los resultados del código: Inferioridad de oportunidades}

Las mujeres con discapacidad física y sensorial tienen menores oportunidades para trabajar que las mujeres en general, debido a que sus oportunidades descienden cuando en un trabajo no hay adaptaciones técnicas para que ellas puedan desarrollar el empleo como cualquier otra persona: no tienen igualdad de oportunidades.

Cuadro 6

\begin{tabular}{|c|c|}
\hline \multirow{4}{*}{$\begin{array}{c}\text { Inferioridad de } \\
\text { oportunidades }\end{array}$} & $\begin{array}{c}\text { Media de } \\
\text { frecuencia } \\
\end{array}$ \\
\cline { 2 - 2 } & 1.51 \\
\hline & Porcentaje \\
& $151.02 \%$ \\
\hline
\end{tabular}

Feminismo/s 13, junio 2009, pp. 153-170 
El porcentaje de frecuencia de este código, al cual hacen referencia las mujeres entrevistadas en sus narrativas, es muy alto y conviene subrayar su significatividad. Aluden a la falta de sensibilización y de adaptaciones técnicas que, al no ser tenidas en cuenta, les obligan a enfrentarse en demasiadas ocasiones en desigualdad. Más en el ámbito laboral, pero también en el educativo y en el familiar.

Los otros trabajos sí había diferencia, porque yo ahora digo «me siento cansada por mi deficiencia, que soy más lenta, que me ahogo» y me dicen descanse o haga otra cosa, pero en los otros trabajos por lo menos en el calzado no te puedes sentar porque te regañan no puedes ir al baño, no tienen en cuenta que tienes una discapacidad. (Fis006)

Sí, eso está claro, la falta de sensibilización y de mentalización de los empresarios y de todas las personas que no conocen a las personas sordas, eso es lo principal, primero deberían de conocernos y darnos una oportunidad y saber que necesitamos que se nos adapten algunas para poder estar en las mismas condiciones que las demás personas, mientras esto no se haga estaremos en inferioridad de condiciones. (Sord007)

La verdad que los empleos son muy diferentes, la mayoría de las mujeres sordas trabajan en la limpieza, los hombres tienen más oportunidades no sólo en la limpieza o las lavanderías como las mujeres, también en la once vendiendo el cupón o en el calzado pero como tienen fuerza pueden trabajar en más sitios, la mujer es más débil, entonces el hombre tiene más oportunidades para encontrar empleo. Los hombres tienen más trabajo para trabajar que la mujer, yo no lo he visto o si lo he visto, ha sido muy poco, a mujeres vendiendo el cupón por la calle a muy pocas y hombres la mayoría, a la mujer le da vergüenza. (Sord002)

Seguro que nos ponen muchas más pegas a nosotras que a cualquier otra mujer, eso está claro. (Fis016)

El mayor problema va a ser el laboral, en ese aspecto es donde la mujer con discapacidad va tener mas problemas. (Vis012)

\subsection{Discusión de los resultados del código: Discriminación por razón de género}

Las mujeres con discapacidad física y sensorial, fundamentalmente, sienten que son discriminadas y tratadas de diferente manera, exclusivamente por el hecho de tener una discapacidad y ser mujeres. 


\section{Cuadro 7}

\begin{tabular}{|c|c|}
\hline \multirow{4}{*}{$\begin{array}{c}\text { Discriminación por } \\
\text { género }\end{array}$} & Media de \\
& frecuencia \\
& 1.71 \\
\cline { 2 - 2 } & Porcentaje \\
& 171.42 \\
\hline
\end{tabular}

El porcentaje de frecuencia de este código es muy elevado, y tiene gran relevancia para las mujeres entrevistadas, comprobamos cómo de las desigualdades entre hombres y mujeres siguen presentes en el mundo laboral y aumentan cuando se suma una discapacidad física y sensorial al rol de mujer:

Pero sí es verdad que las mujeres con discapacidad tienen más dificultades para encontrar un empleo y además en casi todos los trabajos prefieren hombres, que ellos piensan que son mejores y por eso los prefieren, piensan que las mujeres somos peores para trabajar fuera de la casa. (Sord012)

Yo pienso que los trabajos domésticos. Yo pienso que los hombres son más exteriores por lo que tienen más posibilidades de trabajar que la mujer con discapacidad. La mujer busca más trabajar en la casa, con niños. (Fis006)

Aunque sí, siempre tiene más ventaja el hombre, además hasta en personas que no tiene ninguna discapacidad, quien tiene siempre más problemas es la mujer, aunque hoy en día gracias a Dios estamos avanzando, menos mal. (Vis002)

Sí, eso está claro, a los hombres los ven más fuertes y los hombres no tienen que hacer las cosas de la casa, tienen un trabajo menos y para encontrar trabajo yo creo que eso es importante el que no tengas que encargarte de una casa y de hijos, para que te contraten antes. A nosotras nos ven más frágiles y si encima tenemos una deficiencia más todavía, piensan que no vamos a ser capaces o que lo vamos hacer y que vamos a estar pidiendo muchas bajas y eso no lo quiero, cuando eso no tiene por qué ser así, están muy equivocados. (Vis014)

Sí existe desigualdad, yo pienso que al empresario no le importa que el hombre no oiga, eso es lo que pienso yo. Yo imagino que si mi marido no oyera bien para su trabajo, daría igual, él es mecánico, trabaja en un taller, pues así en muchos empleos, yo creo que la mujer aparte de que eres mujer y para muchos trabajos no te quieren, pues si encima tienes una discapacidad, ya sí que no te quieren. (Sord016)

Entonces una persona que le pueda rendir menos que otra pues no la van a contratar teniendo a otra que lo hace más rápido y si encima es mujer no la van a contratar, encima se puede quedar embarazada, por lo que pienso que sí hay una mayor discriminación para encontrar empleo en las mujeres con discapacidad. (Fis004).

Sus oportunidades se reducen, fundamentalmente, por ser mujeres y tener una discapacidad física y sensorial, expresan que el hombre sigue estando por 
encima de ellas para acceder a un empleo. Igualmente, el abanico de trabajos al que pueden optar es mayor para los hombres con o sin ningún tipo de discapacidad y para las mujeres estándar.

\section{Conclusiones}

A partir del estudio realizado se hace patente la falta de datos actualizados que describan con rigor la situación, la condición y el número de las mujeres con discapacidad. No queda pues sino denunciar que sin estos datos no se puede mejorar el desarrollo personal y social de estas personas, tal y como demuestra la difícil aplicación de la Ley de Dependencia ${ }^{19}$ en nuestro país.

Conscientes de ello, exponemos las conclusiones más relevantes de nuestro trabajo.

Las mujeres con discapacidad física y sensorial en un alto porcentaje están condicionadas a ejercer el rol que se les ha impuesto como mujeres. En muchas ocasiones reconocen tener más dificultades para acceder al mundo laboral que los hombres con discapacidad y que las mujeres sin discapacidad. Son pocas las entrevistadas que verbalizan cómo la escasa formación que tienen les perjudica en su inserción laboral y cómo el mismo hecho consolida sus vidas dentro del ámbito privado.

Desde la perspectiva personal, asumen con resignación que la consecuencia natural de ser mujer y tener discapacidad es no acceder a un puesto de trabajo remunerado. Afirman sentirse poco reconocidas a nivel familiar, laboral y social. Nos parece importante subrayar su percepción negativa respecto al modo en que ellas mismas no se ajustan a los estereotipos de su sexo.

Dicha percepción es consecuencia de la proyección social que reciben, basada fundamentalmente en la ignorancia y en la falta de sensibilidad y adaptaciones técnicas y curriculares a su condición. Perciben la inferioridad de oportunidades a la hora de expresar, desarrollar y proyectar sus capacidades. Es interesante que manifiesten ser plenamente sabedoras de su doble discriminación, por razón de género y discapacidad, y que comiencen a reivindicar formación y adaptaciones. Apuntan la discriminación existente, la escasez de trabajo o los problemas para encontrarlo, su precariedad o inadecuación a las particularidades asociadas a cada discapacidad.

19. El 30 noviembre de 2006, el Pleno del Congreso aprobó el Proyecto de Ley de Promoción de la Autonomía Personal y Atención a Personas en Situación de Dependencia (Ley de Dependencia). Esta ley configurará el Sistema de Autonomía y Atención a la Dependencia (SAAD) como cuarto pilar del Estado del Bienestar, tras el Sistema Nacional de Salud, el sistema educativo y el sistema de pensiones, que fueron desarrollados en la década de los ochenta. 
Están realizando un doble esfuerzo en sus trabajos para poder alejar los estereotipos que las victimizan.

\section{Referencias bibliográficas}

Aguado, A. L. Historia de las deficiencias. Madrid, Escuela libre, 1995.

ADroHer, S. Discapacidad e integración, familia, trabajo y sociedad. Madrid, Comillas, Universidad Pontificia, 2004.

CASADO, R. y DE JUAN, N. Inclusión y no discriminación de la mujer discapacitada. Burgos, Universidad de Burgos, 2005.

CROW, L. «Nuestra vida en su totalidad: renovación del modelo social de discapacidad». En Morris, J. (ed). Encuentros con desconocidas. Madrid, Narcea 1996, pp. 22-25.

FERNÁNDEZ, D. Cultura empresarial: motivaciones de los empresarios para la inclusión laboral de personas con discapacidad. A Coruña, Fundación Paidea, 1995.

FERNÁNDEZ, S. Transición, escuela y empleo de las personas con discapacidad. Madrid, MTAS, 2004.

FERnÁndeZ, J (coor.). Genero y sociedad. Madrid, Pirámide, 1998.

GARCía, J. J. y ZARCO, J. El espejo social de la mujer con gran discapacidad. Madrid, Fundamentos, 2004.

García, J. M. y García, N. Empleo y Discapacidad. Madrid, IMSERSO, 1998.

Goffman, I. Estigma. Buenos Aires, Amorrortu, 1980.

HeRnANDO, A. La construcción de la subjetividad femenina. Madrid, Universidad Complutense, Instituto de investigaciones feministas, 2000.

HERNÁNDEZ, I. (coord.). Situación laboral de las mujeres con discapacidad. Madrid, Instituto de la mujer, 2005.

Huber, G. L., Fernández, G., Lorenzo, O y Herrera, L. Análisis de datos cualitativos con Aquad cinco para Windows. Granada, Grupo editorial universitario, 2001.

INSS. Transición escuela-empleo de las personas con discapacidad. Madrid, Autor, 2003.

IMSERSO. Mujer y discapacidad: buenas prácticas. Madrid, Autor, 2000.

IZQuierdo, J. Del Río, O y RodríGuez, A. La Desigualdad de las mujeres en el uso del tiempo. Bilbao, Instituto de la Mujer, 1998.

JimÉnez, A y Huete, A. La discriminación por motivos de discapacidad. Madrid, CERMI, 2002.

JULIANO, D. Excluidas y marginadas una aproximación antropológica. Madrid, Cátedra, 2004.

KEITH, L. Encuentros con personas extrañas: reacciones ante las mujeres discapacitadas. En Jenny, M. (Ed). Encuentros con desconocidas. Madrid, Narcea, 1996, pp. 87-107. 
Lloyd, M. «Does She Boil Eggs? Toward a Feminist Model of Disability». Disability, Handicap and Society, 7, 3 (1992).

NASH, M. y TAVERA, S. Experiencias desiguales. Conflictos sociales y respuestas colectivas (siglo XIX). Madrid, Síntesis, 1994.

Martínez, Ma C. Análisis psicosocial del prejuicio. Madrid, Síntesis, 1996.

MARTínEZ, R. Discapacidad visual: desarrollo, comunicación e intervención. Granada, Grupo editorial universitario, 2005.

MARTíneZ, M. A. y SAULEDA, N. Las narrativas de los profesores. Una perspectiva situada. Alicante, Club Universitario, 2002.

Maruani, M. Trabajo y empleo de las mujeres. Madrid, Fundamentos, 2002.

Morris, J. Encuentros con desconocidas. Madrid, Narcea,1996.

Peláez NarvaÉz, A. M. Maternidad y discapacidad. Madrid, Cermi, Barclays, 2009.

Pereda, C., Prada, M. A., y Actis, W. Discapacidad y Trabajo en España. Madrid, IMSERSO, 1998.

RiU, P. «Una perspectiva de género desde la no estandarización». En Shum, G y Conde, A. (Eds). Las olvidadas: Mujeres en riesgo de exclusión social. Huelva, Universidad, 2006, pp. 186-246.

Ruiz, P. La situación laboral de las mujeres con discapacidad. Madrid, Instituto de la mujer, 2005.

Ruiz, J. I. (2º edición). Metodología de la investigación cualitativa. Bilbao, Universidad de Deusto, 1999

SAltzMAn, J. Equidad y Género. Una teoría integrada de estabilidad y cambio. Madrid, Cátedra /Feminismos, 1994.

Shum, G., CONDE, A. y PORTILlo, I. Mujer, discapacidad y violencia. El rostro oculto de la desigualdad. Madrid, Instituto de la Mujer, 2006.

SHUM, G. Las olvidadas: mujeres en riesgo de exclusión. Huelva, Universidad, 2006.

Sierra, R. Técnicas de investigación social. Teoría y ejercicios. Madrid, Thomson, 2003.

TAYLOR, S. J. y BOGDAN, R. Introducción a los métodos cualitativos de investigación. Buenos Aires, Paidos, 1986.

Verdugo, M. A. De Borja, F. y De Urries, J. (Coors.). Hacia una nueva concepción de la discapacidad. Salamanca, Universidad, 1999.

Villagómez, E. y Martínez, M. I. Adecuación de puestos de trabajo para personas con discapacidad: repercusiones económicas y sociales. Madrid, Observatorio de la discapacidad, 2001.

ZIJDEL, L. «Manifiesto de las mujeres con discapacidad en Europa». En García, N. y Cuesta, Z. (Eds). Minusval. Una aproximación al tema de la discapacidad. Madrid, IMSERSO, 2000, pp 430-431. 